\title{
THE HOMOTOPY TYPE OF CERTAIN LAMINATED MANIFOLDS
}

\author{
R. J. DAVERMAN AND F. C. TINSLEY
}

\begin{abstract}
Let $M$ denote a connected $(n+1)$-manifold $(n \geq 5)$. A lamination $G$ of $M$ is an usc decomposition of $M$ into closed connected $n$-manifolds. Daverman has shown that the decomposition space $M / G$ is homeomorphic to a 1-manifold possibly with boundary. If $M / G=R^{1}$, we prove that $M$ has the homotopy type of an $n$-manifold if and only if $\Pi_{1}(M)$ is finitely presented. In the case that $M / G=S^{1}$ we use the above result to construct an approximate fibration $f: M \rightarrow S^{1}$. We then discuss the important interactions of this study with that of perfect subgroups of finitely presented groups.
\end{abstract}

0. Introduction. Let $M$ be an $(n+1)$-manifold. A lamination of $M$ is an usc decomposition $G$ of $M$ into closed connected $n$-manifolds. The decomposition space $M / G$ is always a 1-manifold, possibly with boundary [4, Theorem 3.3]. The main theorem concerns the case $M / G=R^{1}$. We show that $M$ is homotopy equivalent to a closed codimension one submanifold if and only if its fundamental group $\Pi_{1}(M)$ is finitely presented. Finally, we apply this theorem to the case where $M / G=S^{1}$ to find an approximate fibration $f: M \rightarrow S^{1}$. As a corollary, if the Whitehead group $\operatorname{Wh}\left(\Pi_{1}(M)\right)$ is trivial, then $M$ is a locally trivial fibration over $S^{1}$.

The roots of this research spring from a more general investigation of decompositions of manifolds into closed connected manifolds of arbitrary fixed codimension. A detailed discussion of this investigation appears in a survey article by Daverman [5].

1. Statement of results. We state the main theorem and prove its corollaries. In this section $M$ will always denote an $(n+1)$-manifold with $n \geq 5, G$ an usc decomposition of $M$ into closed connected $n$-manifolds, and $p: M \rightarrow M / G$ the decomposition map.

THEOREM 1.1. Suppose $M$ admits a lamination $G$ with $M / G=R^{1}$. Then $M$ admits another lamination $G^{\prime}$ with a locally flat element $g^{\prime} \in G^{\prime}$ such that the inclusion $i: g^{\prime} \rightarrow M$ is a homotopy equivalence if and only if $\Pi_{1}(M)$ is finitely presented.

This theorem gives information about other laminated manifolds.

COROLLARY 1.2. Suppose $M$ admits a lamination $G$ with $M / G=S^{1}$. Then there is an approximate fibration $f: M \rightarrow S^{1}$.

Received by the editors February 15, 1985. Presented at the 90th Annual Meeting of the Society, Louisville, Kentucky, on January 27, 1984.

1980 Mathematics Subject Classification. Primary 57N15; Secondary 55P15.

Key words and phrases. Lamination, decomposition, approximate fibration, homotopy type, finitely presented group, perfect subgroup. 
COROLlary 1.3. Suppose $M$ admits a lamination $G$ with $M / G=S^{1}$ and $\mathrm{Wh}\left(\Pi_{1}(M)\right)=0$. Then $M$ is a locally trivial fibration over $S^{1}$.

PROOF OF COROLlaRIES 1.2 AND 1.3. Choose $g \in G$ locally flat $[4$, Lemma 6.1]. Then the complement of $g, M-g$, inherits the lamination $G-g$ from $G$. Since $g$ is locally flat, the manifold $M-g$ deformation retracts to a compact manifold (in particular, a compact ENR). As a result $M-g$ is dominated by a finite CWcomplex $X$. The group $\Pi_{1}(M-g)$ is therefore a retract of the finitely presented group $\Pi_{1}(X)$ and as such is itself finitely presented [17, Lemma 1.3]. Also the decomposition space $(M-g) /(G-g)$ is homeomorphic to $R^{1}$. Using Theorem 1.1 relaminate $M-g$ with a lamination $G^{\prime}$ having an element $g^{\prime} \in G^{\prime}$ with $i^{\prime}: g^{\prime} \rightarrow M-g$ a homotopy equivalence. Now $G^{\prime} \cup\{g\}$ is a lamination of $M[4$, Theorem 3.4]. Let $\alpha: g^{\prime} \times[-1,1] \rightarrow M$ be a bicollar on $g^{\prime}$. Then $M^{\prime}=M-\alpha\left(g^{\prime} \times(0,1 / 2)\right)$ is an $h$-cobordism. There is a homeomorphism $h: M^{\prime}-\alpha\left(g^{\prime} \times 1 / 2\right) \rightarrow g^{\prime} \times[0,1)$ with $h(\alpha(z, 0))=(z, 0)\left[\mathbf{1 4}\right.$, Corollary 4.13.1]. Let $G^{\prime \prime}=\left\{\alpha\left(g^{\prime}, s\right), h^{-1}\left(g^{\prime} \times t\right), 0<\right.$ $s \leq 1 / 2,0 \leq t<1\}$. Then $G^{\prime \prime}$ is a lamination of $M[4$, Theorem 3.4]. Finally $p^{\prime \prime}: M \rightarrow M / G^{\prime \prime}=S^{1}$ is an approximate fibration $[9$, Theorem C]. This proves Corollary 1.2 . If $\mathrm{Wh}\left(\Pi_{1}(M)\right)$ vanishes, then $p^{\prime \prime}$ is homotopic to a locally trivial fibration [9, Corollary F].

2. Proof of the Main Theorem. We introduce the following notation. Suppose $M$ admits a lamination $G$ with $M / G=R^{1}$. Consequently, $M$ has precisely two ends. Let $N$ be any closed $n$-manifold which separates those ends. We denote by $N^{+}$and $N^{-}$the closures of the components of $M-N$. One implication in the Main Theorem is easy since a compact manifold has finitely presented fundamental group (see the proofs of 1.2 and 1.3 above). For the other we suppose $\Pi_{1}(M)$ is finitely presented. Assume $M$ has a PL triangulation.

Step I. Since $\Pi_{1}(M)$ is finitely presented, we can find a finite 2-complex $P$ in $M$ with inclusion inducing an isomorphism on fundamental groups. Choose $g \in G$ locally flat [4, Lemma 6.1] with $g \cap P=\varnothing$ and $P \subset g^{+}$. Let $i: g \rightarrow g^{+}$and $j: g^{+} \rightarrow M$ be inclusions. The induced homomorphism $i_{\#}: \Pi_{1}(g) \rightarrow \Pi_{1}\left(g^{+}\right)$is a surjection [6, Lemma 3.1]. Since $P$ carries $\Pi_{1}(M), j_{\#}: \Pi_{1}\left(g^{+}\right) \rightarrow \Pi_{1}(M)$ is a surjection. Let $l$ be a loop in $g^{+}$representing an element of $\operatorname{kernel}\left(j_{\#}\right)$. Since $i_{\#}$ is surjective, $l$ is freely homotopic in $g^{+}$to a loop $l^{\prime}$ in $P$. By construction, $l^{\prime}$ must bound a disk in $P$. Thus, $\operatorname{kernel}\left(j_{\#}\right)=1$ and $j_{\#}$ is an isomorphism.

Step II. Since $\Pi_{1}\left(g^{+}\right)$and $\Pi_{1}(g)$ are both finitely presented, the kernel of $i_{\#}$ : $\Pi_{1}(g) \rightarrow \Pi_{1}\left(g^{+}\right)$is the normal closure of a finite set $S$ of cardinality, say, $m[\mathbf{1 5}$, Lemma 3.11]. Now $n \geq 5$ so we can attach $m$ mutually disjoint, PL embedded 2-handles in $g^{+}$to $g$ with the cores of the attaching regions corresponding to representatives of the set $S$. We denote these handles by $h_{i}^{2}, 1 \leq i \leq m$. These surgeries yield a new manifold pair $\left(N_{1}^{+}, N_{1}\right)$ with $N_{1}$ the boundary of $N_{1}^{+}$and the inclusion inducing an isomorphism of fundamental groups.

Step III. Denote the universal cover of $M$ by $\tilde{M}$. For any subset $U$ of $M, \tilde{U}$ will denote the preimage of $U$ in $\tilde{M}$.

By pulling back the lamination of $M, \tilde{M}$ has a partition $\{\tilde{g} \mid g \in G\}$ which may fail to be a lamination only because it may not be usc. However, it is still the case that for any $\tilde{g}, H_{*}\left(\widetilde{g^{+}}, \tilde{g}\right)=0[4$, Lemma 6.2$]$. 
Let $h^{2}=\bigcup_{i=1}^{m} h_{i}^{2}$. Consider the triple $\left(\widetilde{g^{+}}, \widetilde{g \cup h^{2}}, \tilde{g}\right)$. Note that $\widetilde{g^{+}}$and $\widetilde{g \cup h^{2}}$ are the universal covers of $g^{+}$and $g \cup h^{2}$ respectively. The exact homology sequence of this triple follows:

$$
\rightarrow H_{k}\left(\widetilde{g \cup h^{2}}, \tilde{g}\right) \rightarrow H_{k}\left(\widetilde{g^{+}}, \tilde{g}\right) \rightarrow H_{k}\left(\widetilde{g^{+}} \widetilde{g \cup h^{2}}\right) \rightarrow
$$

By the above, $H_{*}\left(\widetilde{g^{+}}, \tilde{g}\right)=0$. Thus, the boundary-induced homomorphism $\partial_{*}$ : $H_{k+1}\left(\widetilde{g^{+}}, \widetilde{g \cup h^{2}}\right) \rightarrow H_{k}\left(\widetilde{g \cup h^{2}}, \tilde{g}\right)$ is an isomorphism. By excision, $H_{k+1}\left(\widetilde{g^{+}}, \widetilde{g \cup h^{2}}\right)=$ $H_{k+1}\left(\widetilde{N_{1}^{+}}, \widetilde{N_{1}}\right)$. Each 2-handle $h_{i}^{2}$ is isomorphic to $D_{i}^{2} \times D_{i}^{n-1}$ with $g \cap h_{i}^{2}=$ $S_{i}^{1} \times D_{i}^{n-1}$.

Again by excision,

$$
\begin{aligned}
H_{*}\left(\widetilde{g \cup h^{2}}, \tilde{g}\right) & =H_{*}\left(\widetilde{\bigcup_{i=1}^{m} D^{2} \times D^{n-1}}, \widetilde{\bigcup_{i=1}^{m} S^{1} \times D^{n-1}}\right) \\
& =\bigoplus_{i=1}^{m} H_{*}(\widetilde{D_{i}^{2} \times D_{i}^{n-1}}, \overbrace{S_{i}^{1} \times D_{i}^{n-1}}) .
\end{aligned}
$$

Therefore viewed as a $Z \Pi_{1}$-module

$$
H_{k}\left(\widetilde{g \cup h^{2}}, \tilde{g}\right)= \begin{cases}\bigoplus_{i=1}^{m} Z \Pi_{1}, & k=2, \\ 0, & k \neq 2 .\end{cases}
$$

Step IV. From Step III we have that

$$
H_{k}\left(\widetilde{N_{1}^{+}}, \widetilde{N_{1}}\right)= \begin{cases}\bigoplus_{i=1}^{m} Z \Pi_{1}, & k=3, \\ 0, & k \neq 3 .\end{cases}
$$

Both $\widetilde{N_{1}^{+}}$and $\widetilde{N_{1}}$ are simply connected. Thus, the Hurewicz Theorem [14, p. 399] yields:

$$
\Pi_{k}\left(\widetilde{N_{1}^{+}}, \widetilde{N_{1}}\right)= \begin{cases}\bigoplus_{i=1}^{m} Z \Pi_{1}, & k=3, \\ 0, & k<3 .\end{cases}
$$

Also $\Pi_{k}\left(\widetilde{N_{1}^{+}}, \widetilde{N_{1}}\right)=\Pi_{k}\left(N_{1}^{+}, N_{1}\right)$. So for each $i, 1 \leq i \leq m$, we may choose a representative of a generator $f_{i}:\left(D^{3}, S^{2}\right) \rightarrow\left(N_{1}^{+}, N_{1}\right)$.

If $\operatorname{dim}(M)=n+1 \geq 7$ we may assume that these are mutually disjoint PL embeddings (by general position). For the case $n=5$ see the Appendix. For each $i, 1 \leq i \leq m$, we attach a 3 -handle $h_{i}^{3}$ with $f_{i}\left(D^{3}\right)$ as its core. We may assume that this collection of 3 -handles is mutually disjoint. These surgeries yield a new manifold pair $\left(N_{2}^{+}, N_{2}\right)$ with $N_{2}$ the boundary of $N_{2}^{+}$.

Step V. Let $h^{3}=\bigcup_{i=1}^{m} h_{i}^{3}$. Consider the exact homology sequence of the triple $\left(\widetilde{N_{1}^{+}}, \widetilde{N_{1} \cup h^{3}}, \widetilde{N_{1}}\right)$ :

$$
\rightarrow H_{k}\left(\widetilde{N_{1} \cup h^{3}}, \widetilde{N_{1}}\right) \rightarrow H_{k}\left(\widetilde{N_{1}^{+}}, \widetilde{N_{1}}\right) \rightarrow H_{k}\left(\widetilde{N_{1}^{+}}, \widetilde{N_{1} \cup h^{3}}\right) \rightarrow
$$

By excision, $H_{*}\left(\widetilde{N_{1}^{+}}, \widetilde{N_{1} \cup h^{3}}\right)=H_{*}\left(\widetilde{N_{2}^{+}}, \widetilde{N_{2}}\right)$. By construction

$$
i_{*}: H_{k}\left(\widetilde{N_{1} \cup h^{3}}, \widetilde{N_{1}}\right) \rightarrow H_{k}\left(\widetilde{N_{1}^{+}}, \widetilde{N_{1}}\right)
$$

is an isomorphism for all $k$. Thus, $H_{*}\left(\widetilde{N_{2}^{+}}, \widetilde{N_{2}}\right)=0$ so $\Pi_{*}\left(\widetilde{N_{2}^{+}}, \widetilde{N_{2}}\right)=0$ and $\Pi_{*}\left(N_{2}^{+}, N_{2}\right)=0$. 
Step VI. A similar argument can be given to show that $\Pi_{*}\left(N_{2}^{-}, N_{2}\right)=0$. However, this follows from the fact that the cobordism bounded by $g$ and $N_{2}$ is precisely that obtained from the plus construction of Quillen [12]. As a result, the inclusion $i: N_{2} \rightarrow M$ is a homotopy equivalence.

Step VII. Finally we construct a new lamination $G^{\prime}$ of $M$ with $N_{2} \in G^{\prime}[\mathbf{6}$, Theorem 4.2]. Let $g^{\prime}=N_{2}$.

This completes the proof of Theorem 1.1 in the case that $M$ is PL. However, since $n \geq 5$ this argument can be generalized to topological manifolds [11, Essay III].

3. The fundamental group of a laminated manifold $M$. In light of Theorem 1.1 we pose the following question:

Question 3.1. Suppose $M$ admits a lamination $G$ with $M / G=R^{1}$. Must $\Pi_{1}(M)$ be finitely presented?

Suppose the answer is negative. Let $g_{t}=p^{-1}(t) \subset M$ for $t \in R^{1}$. Since $i: g_{0} \rightarrow M$ induces a surjection on fundamental groups [6, Lemma 3.1] and the kernel of this induced homomorphism is perfect $\left[\mathbf{7}\right.$, Lemma 2.4], then $\Pi_{1}\left(g_{0}\right)$ would be a finitely presented group with a perfect normal subgroup which is not the normal closure of a finite set. Question 3.1 is equivalent to an apparently quite difficult problem in combinatorial and homological group theory.

DEFINITION 3.2. A group $G$ is almost finitely presented if there exists a short exact sequence $0 \rightarrow R \rightarrow F \rightarrow G \rightarrow 0$ with $F$ finitely generated and free and $R / R^{\prime}$ finitely generated as a $Z G$ module ( $R^{\prime}$ is the commutator subgroup of $R$ ).

Question 3.3 . Is every almost finitely presented group $G$ finitely presented $[\mathbf{1 8}$, Problem F10, p. 387]?

The existence of a laminated $M$ with a nonfinitely presented fundamental group is slightly more restrictive. Denote by $N_{t}$ the closure of the bounded component of $M-\left\{g_{0} \cup g_{t}\right\}$. Then the inclusion of $g_{0}$ into $N_{t}$ induces a homomorphism of finitely presented groups with perfect kernel for $t>0$. The existence of such a laminated $M$ would imply a positive answer to the following question.

Question 3.4. Does there exist a finitely presented group $G$ with perfect normal subgroup $P$ such that

(1) $P=\bigcup_{i=1}^{\infty} P_{i}$,

(2) $P_{i} \subset P_{i+1}$ for all $i$,

(3) each $P_{i}$ is perfect and is the normal closure of a finite set, and

(4) $P$ is not the normal closure of a finite set?

In each of the laminated manifolds constructed by us the kernel $P_{i}$ is actually the normal closure of a finitely generated perfect group [7]. Thus, we also ask

Question 3.5. Does there exist a finitely presented group $G$ with perfect normal subgroup $P$ such that

(1) same as in 3.4 ,

(2) same as in 3.4,

(3) each $P_{i}$ is the normal closure in $G$ of a finitely generated perfect group,

(4) same as in 3.4 ?

Appendix. We complete Step IV of the proof of Theorem 1.1 for the case $n=5$. Recall that $\Pi_{3}\left(N_{1}^{+}, N_{1}\right)$ is a free $Z \Pi_{1}$ module on $m$ generators $\left\{f_{i}:\left(D^{3}, S^{2}\right) \rightarrow\right.$ $\left.\left(N_{1}^{+}, N_{1}\right) \mid 1 \leq i \leq m\right\}$. Since the dimensions of $D^{3}$ and $N_{1}$ are 3 and 6 respectively 
and $\Pi_{k}\left(N_{1}^{+}, N_{1}\right)=0$ for $k<3$, we may assume that the $f_{i}$ 's are PL embeddings $[8$, Corollary 8.2]. However, they may not be mutually disjoint. We show how to remove the intersections using the Whitney Lemma [13, Lemma 5.12].

For each $i$ denote $\left(f_{i}\left(D^{3}\right), f_{i}\left(S^{2}\right)\right)$ by $\left(D_{i}^{3}, S_{i}^{2}\right)$. By general position we may assume that any pair of these 3 -disks intersect in their interiors in at most finitely many points distinct from the intersection points of any other pair.

Suppose $x \in\left(D_{1}^{3} \cap D_{2}^{3}\right) \subset\left(N_{1}^{+}-N_{1}\right)$. We show how to eliminate this intersection without disturbing any other existing intersection points or introducing any new intersection points. The result then follows by induction.

Choose PL arcs $\alpha_{1}$ and $\alpha_{2}$ joining $x$ to $S_{1}^{2}$ and $S_{2}^{2}$ respectively. We may assume $\alpha_{i}$ misses other intersection points and int $\left(\alpha_{i}\right) \cap\left\{S_{i}^{2} \cup x\right\}=\left\{x_{i} \cup x\right\}$, where $x_{i} \in S_{i}^{2}$. Since the inclusion of $N_{1}$ into $N_{1}^{+}$induces an isomorphism on fundamental groups, there is an arc $\beta$ joining $x_{1}$ and $x_{2}$ in $N_{1}$ so that the simple closed curve $\gamma=\alpha_{2} \alpha_{1}^{-1} \beta$ is trivial in $N_{1}^{+}$and $\gamma \cap S_{j}^{2}=\varnothing$ for $j>2$. Let $B$ be a PL 2-disk bounded by $\gamma$ with $B \cap\left\{N_{1} \cup D_{1}^{3} \cup D_{2}^{3}\right\}=\gamma$ and $B \cap D_{j}^{3}=\varnothing$ for $j>2$.

For the following we use [13, Chapter 5] as a general reference. Let $\left(L, L_{1}, L_{2}\right)$ be a regular neighborhood of $B$ in $\left(N_{1}^{+}, D_{1}^{3}, D_{2}^{3}\right)$. Then each $\left(L, L_{i}\right)$ is an unknotted ball pair. However, $\operatorname{bdy}\left(L_{1}\right)$ and $\operatorname{bdy}\left(L_{2}\right)$ are linked in $\operatorname{bdy}(L)$. For each $i$, $\operatorname{bdy}\left(L_{i}\right) \cap N_{1}=E_{i}$, a PL 2-ball. Also $\beta$ joins $\operatorname{int}\left(E_{1}\right)$ to $\operatorname{int}\left(E_{2}\right)$.

By an isotopy of $N_{1}$ with support in an arbitrary neighborhood of $\beta$ we "push $\operatorname{int}\left(E_{1}\right)$ through $\operatorname{int}\left(E_{2}\right)$ along $\beta$ " with $E_{1}$ and $E_{2}$ oriented so as to unlink bdy $\left(L_{1}\right)$ and bdy $\left(L_{2}\right)$. We extend this isotopy to $N_{1}^{+}$using a small collar on $N_{1}$.

The effect of this isotopy is to introduce an intersection $x^{\prime}$ of $D_{1}^{3}$ and $D_{2}^{3}$ with $\epsilon\left(x^{\prime}\right)=-\epsilon(x)$, where $\epsilon(x) \in Z \Pi_{1}$ is the standardly defined intersection number. Now the Whitney Lemma applies to remove both $x$ and $x^{\prime}$ as intersections.

\section{REFERENCES}

1. K. S. Brown, Cohomology of groups, Springer-Verlag, Berlin and New York, 1982.

2. R. Bieri and R. Strebel, Metaabelian quotients of finitely presented soluable groups are finitely presented, Homological Group Theory (C. T. C. Wall, ed.), Cambridge Univ. Press, London, 1979, pp. 231-234.

3. M. M. Cohen, A course in simple homotopy theory, Springer-Verlag, Berlin and New York, 1973.

4. R. J. Daverman, Decompositions of manifolds into codimension one submanifolds, Compositio Math. 55 (1985), 185-207.

5. __ Decompositions into submanifolds of fixed codimension, manuscript.

6. R. J. Daverman and F. C. Tinsley, Laminated decompositions involving a given submanifold, Topology Appl. 20 (1985), 107-119.

7. __ Laminations, finitely generated perfect groups, and acyclic maps, preprint.

8. J. P. F. Hudson, Piecewise linear topology, Math. Lecture Notes Series, Benjamin, New York, 1969.

9. L. S. Husch, Approximating approximate fibrations by fibrations, Canad. J. Math. 29 (1977), 897-913.

10. _ Lecture notes, Univ. of Tennessee.

11. R. C. Kirby and L. C. Siebenmann, Foundational essays on topological manifolds, smoothings, and triangulations, Ann. of Math. Stud., No. 88, Princeton Univ. Press, Princeton, N. J., 1977.

12. D. Quillen, Cohomology of groups, Actes Congres Internat., Vol. 2, 1970, pp. 47-51.

13. C. P. Rourke and B. J. Sanderson, Introduction to piecewise-linear topology, SpringerVerlag, Berlin and New York, 1972. 
14. T. B. Rushing, Topological embeddings, Pure and Appl. Math., Vol. 52, Academic Press, New York, 1973.

15. L. C. Siebenmann, Ph.D thesis, Princeton Univ., 1965.

16. E. H. Spanier, Algebraic topology, McGraw-Hill, New York, 1966.

17. C. T. C. Wall, Finiteness conditions for $C W$-complexes, Proc. Royal Soc. London Ser. A 295 (1966), 129-139.

18. _ (editor), Homological group theory, Cambridge Univ. Press, London, 1979.

Department of Mathematics, University of Tennessee, Knoxville, TenNESSEE 37996-1300

Department of Mathematics, The Colorado College, Colorado Springs, COLORADO 80903 